\title{
Odd Jacobi Manifolds and Loday-Poisson Brackets
}

\author{
Andrew James Bruce \\ Institute of Mathematics, Polish Academy of Sciences, Śniadeckich 8, P.O. Box 21, 00-956 Warszawa, Poland \\ Correspondence should be addressed to Andrew James Bruce; andrewjamesbruce@googlemail.com
}

Received 24 December 2013; Accepted 18 February 2014; Published 7 April 2014

Academic Editor: Luis J. Alias

Copyright (C) 2014 Andrew James Bruce. This is an open access article distributed under the Creative Commons Attribution License, which permits unrestricted use, distribution, and reproduction in any medium, provided the original work is properly cited.

We construct a nonskew symmetric version of a Poisson bracket on the algebra of smooth functions on an odd Jacobi supermanifold. We refer to such Poisson-like brackets as Loday-Poisson brackets. We examine the relations between the Hamiltonian vector fields with respect to both the odd Jacobi structure and the Loday-Poisson structure. Furthermore, we show that the Loday-Poisson bracket satisfies the Leibniz rule over the noncommutative product derived from the homological vector field.

In memory of Jean-Louis Loday (1946-2012)

\section{Introduction}

In this paper, we construct a Loday-Poisson bracket on the algebra of smooth functions on an odd Jacobi supermanifold $(M, S, Q)$; see [1]. The key observation here is that the homological vector field $Q \in \operatorname{Vect}(M)$ is a Jacobi vector field with respect to the odd Jacobi structure. Due to this, one can directly employ the notion of derived brackets as defined by Kosmann-Schwarzbach [2,3]. We will draw heavily from these works (the notion of a derived bracket can be traced back to J. L. Koszul (1990) in unpublished notes. The notion was also put forward by Th. Th. Voronov (1993), but remains unpublished).

On any differential super Lie algebra, $(A,[], D$,$) , with$ bracket of even or odd parity, one can construct the "derived bracket"

$$
(a, b)_{D}:=(-1)^{\tilde{a}+1}[D(a), b] .
$$

This bracket is not in general a Lie bracket (or a Lie antibracket) due to the lack of skew symmetry. However, the bracket does satisfy a form of the Jacobi identity. Such Lie algebra mod, the skew symmetry was first introduced by Loday under the name "Leibniz algebras" [4, 5]; we will shortly be a little more precise here.

On an odd Jacobi manifold, we have a differential Lie antialgebra, that is, an odd Lie bracket provided by the odd
Jacobi bracket and a differential provided by the homological vector field.

In essence on an odd Jacobi manifold, one also has "a Poisson bracket mod the skew symmetry." We make the previous statement more precise and then investigate the relations between the Hamiltonian vector fields given by the odd Jacobi and the Loday-Poisson structure. Furthermore, we study the notion of an odd form of noncommutative multiplication on the smooth functions on an odd Jacobi manifold generated by the homological vector field. It is shown that the Loday-Poisson bracket satisfies the right Leibniz rule over both the standard product of smooth functions and the odd derived product.

Remark 1. Grabowski and Marmo in $[6,7]$ prove that on a classical (purely even) manifold $N$ the Jacobi-Loday identity together with the (generalized) Leibniz rule for a binary bracket on $C^{\infty}(N)$ implies the skew symmetry: there can only be Poisson or even Jacobi brackets on classical manifolds. In fact, they show that similar statements also hold for NambuPoisson brackets and for generalizations of Lie algebroid brackets. However, here we consider supermanifolds, meaning that we have a $\mathbb{Z}_{2}$-grading and, more importantly, nontrivial nilpotent functions which invalidate the conditions of their theorem. 
This paper is arranged as follows. In Section 2, we recall the definition of an odd Jacobi manifold and present some results needed throughout the rest of this paper. In Section 3, we construct the Loday-Poisson bracket on an odd Jacobi manifold and, in Section 4, we present some standard examples. The Hamiltonian vector fields with respect to the odd Jacobi brackets and the Loday-Poisson brackets are the subject of Section 5. The derived product generated by a homological vector field is defined and studied in Section 6. Here, it is shown that the Loday-Poisson bracket satisfies an appropriate Leibniz rule over the noncommutative derived product. In Section 7, the previous constructions are applied to the specific case of Jacobi algebroids. We conclude this paper with some final remarks in Section 8. The remainder of the introduction is devoted to some preliminary notions and nomenclature.

1.1. Preliminaries. All vector spaces and algebras will be $\mathbb{Z}_{2}$-graded. We will generally omit the prefix super. By manifold, we will mean a smooth supermanifold. We denote the Grassmann parity of an object by tilde: $\widetilde{A} \in \mathbb{Z}_{2}$. By even or $o d d$, we will be referring explicitly to the Grassmann parity.

A Poisson $(\varepsilon=0)$ or Schouten $(\varepsilon=1)$ algebra is understood as a vector space $A$ with a bilinear associative multiplication and a bilinear operation $\{\cdot, \cdot\}_{\varepsilon}: A \otimes A \rightarrow A$ such that

$$
\begin{aligned}
& \text { Grading } \widetilde{\{a, b\}_{\varepsilon}}=\tilde{a}+\widetilde{b}+\varepsilon ; \\
& \text { Skew symmetry }\{a, b\}_{\varepsilon}=-(-1)^{(\widetilde{a}+\varepsilon)(\tilde{b}+\varepsilon)}\{b, a\}_{\varepsilon} ; \\
& \text { Jacobi identity } \sum_{\text {cyclic }(a, b, c)}(-1)^{(\widetilde{a}+\varepsilon)(\widetilde{c}+\varepsilon)}\left\{a,\{b, c\}_{\varepsilon}\right\}_{\varepsilon}= \\
& 0 ; \\
& \text { Leibniz rule }\{a, b c\}_{\mathcal{\varepsilon}}=\{a, b\}_{\varepsilon} c+(-1)^{(\widetilde{a}+\varepsilon) \tilde{b}} b\{a, c\}_{\varepsilon} .
\end{aligned}
$$

For all homogenous elements $a, b$, and $c \in A$. Extension to inhomogeneous elements is via linearity. If the Leibniz rule does not hold identically but is modified as

$$
\{a, b c\}_{\varepsilon}=\{a, b\}_{\mathcal{\varepsilon}} c+(-1)^{(\widetilde{a}+\varepsilon) \widetilde{b}} b\{a, c\}_{\mathcal{\varepsilon}}-\{a, \mathbb{1}\}_{\mathcal{\varepsilon}} b c
$$

then we have even $(\epsilon=0)$ or odd $(\epsilon=1)$ Jacobi algebras.

A manifold $M$ such that $C^{\infty}(M)$ is a Poisson/Schouten algebra is known as a Poisson/Schouten manifold. In particular, the cotangent of a manifold comes equipped with a canonical Poisson structure.

Let us employ natural local coordinates $\left(x^{A}, p_{A}\right)$ on $T^{*} M$, with $\widetilde{x}^{A}=\widetilde{A}$ and $\widetilde{p}_{A}=\widetilde{A}$. Local diffeomorphisms on $M$ induce vector bundle automorphism on $T^{*} M$ of the form

$$
x^{A^{\prime}}=x^{A^{\prime}}(x), \quad p_{A^{\prime}}=\left(\frac{\partial x^{B}}{\partial x^{A^{\prime}}}\right) p_{B} .
$$

We will in effect use the local description as a natural vector bundle to define the cotangent bundle of a supermanifold. The canonical Poisson bracket on the cotangent is given by

$$
\{F, G\}=(-1)^{\widetilde{A} \widetilde{F}+\widetilde{A}} \frac{\partial F}{\partial p_{A}} \frac{\partial G}{\partial x^{A}}-(-1)^{\widetilde{A} \widetilde{F}} \frac{\partial F}{\partial x^{A}} \frac{\partial G}{\partial p_{A}} .
$$

A manifold equipped with an odd vector field $Q$, such that the nontrivial condition $Q^{2}=(1 / 2)[Q, Q]=0$ holds, is known as a $Q$-manifold. The vector field $Q$ is known as a homological vector field for obvious reasons.

The notion of a Loday-Poisson bracket is important in this paper. We define such a bracket as "a Poisson bracket but without the skewsymmetry." Note that the Jacobi identity, as presented above, for an even bracket (with or without a Leibniz rule) implies that the adjoint endomorphism $f \leadsto$ $\operatorname{ad}_{f}:=\{f, \cdot\}$ is a derivation over the bracket itself. That is,

$$
\operatorname{ad}_{f}\{g, h\}=\left\{\operatorname{ad}_{f}(g), h\right\}+(-1)^{\tilde{f} \tilde{g}}\left\{g, \operatorname{ad}_{f}(h)\right\}
$$

Note that the above has direct meaning even if the skew symmetry of the bracket is weakened. Thus, the most useful definition of the Jacobi identity for an even bracket when the symmetry is lost is

$$
\{f,\{g, h\}\}=\{\{f, g\}, h\}+(-1)^{\tilde{f} \tilde{g}}\{g,\{f, h\}\} .
$$

We refer to this form of the Jacobi identity as the (right) Jacobi-Loday identity [4]. If we have an even bracket that satisfies the Jacobi-Loday identity and the right Leibniz rule, but not necessarily the skew symmetry, then we call such a bracket as a right Loday-Poisson bracket. Left Loday-Poisson brackets can be similarly defined, as can Loday-Poisson brackets that satisfy the Leibniz rule in both directions. For example, classical Poisson brackets are specific examples of Loday-Poisson bracket, albeit skew symmetric. We will examine only right Loday-Poisson brackets, but we will typically drop explicit reference to the word right. To the author's knowledge (noncommutative), Loday-Poisson algebra was first discussed by Casas and Datuashvili [8].

Remark 2. Much of this paper will generalize directly to QSmanifolds in the sense of Voronov [9]. A primary example of a QS-manifold is the antitangent bundle ПTM of a Poisson manifold $M$. The Schouten structure is supplied by the Koszul-Schouten bracket and the homological vector field by the de Rham differential [10]. The associated Loday-Poisson bracket is a natural extension of the Poisson bracket on $C^{\infty}(M)$ to differential forms over $M$, but note this extension is as a Loday bracket only [2]. The constructions here will not directly generalize to even Jacobi supermanifolds due to incompatibility of the Grassmann parities. There is no canonical choice of a homological Jacobi vector field on an even Jacobi supermanifold.

\section{Odd Jacobi Manifolds}

Lichnerowicz $[11,12]$ introduced the notion of a Poisson manifold as well as a Jacobi manifold. Such manifolds have found applications in classical mechanics and play an important role in quantisation. In this section, we recall some of the basic notions as pertaining to odd Jacobi (super) manifolds. No proofs are given here and can be found in [1] or follow directly from the definitions. For a modern discussion of Jacobi structures, including $\mathbb{Z}$-graded versions, see [13-15]. 
Definition 3. An odd Jacobi structure $(S, Q)$ on a manifold $M$ consists of

(i) an odd function $S \in C^{\infty}\left(T^{*} M\right)$, of degree two in fibre coordinates,

(ii) an odd vector field $Q \in \operatorname{Vect}(M)$,

such that the following conditions hold:

(1) the homological condition $Q^{2}=(1 / 2)[Q, Q]=0$,

(2) the invariance condition $L_{Q} S=0$,

(3) the compatibility condition $\{S, S\}=-2 Q \mathcal{Q}$.

Here, $Q \in C^{\infty}\left(T^{*} M\right)$ is the principal symbol or "Hamiltonian" of the vector field $Q$. The brackets $\{\cdot, \cdot\}$ are the canonical Poisson brackets on the cotangent bundle of the manifold.

A manifold equipped with an odd Jacobi structure $(S, Q)$ is known as an odd Jacobi manifold.

Remark 4. At first glance, the above definition seems different from the classical notion of a Jacobi manifold. The compatibility condition involves the principal symbol of the homological vector field rather than the vector field itself. However, all we are really doing is thinking of the vector field as a linear function on the cotangent bundle.

Definition 5. The odd Jacobi bracket on $C^{\infty}(M)$ is defined as

$$
\begin{aligned}
{[f, g]_{J}=} & (-1)^{\tilde{f}+1}\{\{S, f\}, g\}-(-1)^{\tilde{f}+1}\{Q, f g\} \\
= & (-1)^{(\widetilde{B}+1) \tilde{f}+1} S^{B A} \frac{\partial f}{\partial x^{A}} \frac{\partial g}{\partial x^{B}} \\
& +(-1)^{\tilde{f}}\left(Q^{A} \frac{\partial f}{\partial x^{A}}\right) g+f\left(Q^{A} \frac{\partial g}{\partial x^{A}}\right),
\end{aligned}
$$

with $f, g \in C^{\infty}(M)$.

The odd Jacobi bracket makes the algebra of smooth functions on $M$ into an odd Jacobi algebra.

Remark 6. The definition of an odd Jacobi manifold given here is not quite the most general, and one can include an odd function in the construction of an odd Jacobi structure; see Grabowski and Marmo [14] for details. Note that the definition given by Grabowski and Marmo coincide with that given here (up to conventions) up on setting the odd function to zero. Thus, there are examples of odd Jacobi brackets on supermanifolds not covered by the constructions here.

Definition 7. Given a function $f \in C^{\infty}(M)$, the associated Hamiltonian vector field is given by

$$
\begin{gathered}
f \rightsquigarrow X_{f} \in \operatorname{Vect}(M), \\
X_{f}(g)=(-1)^{\tilde{f}}[[f, g]]_{J}-Q(f) g .
\end{gathered}
$$

Note that the homological vector field $Q$ is itself Hamiltonian with respect to the unit constant function: $Q=X_{\mathbb{1}}=$ $[[\mathbb{1}, \cdot]]_{J}$.
Definition 8. A vector field $X \in \operatorname{Vect}(M)$ is said to be a Jacobi vector field if and only if

$$
L_{X} S=\{\chi, S\}=0, \quad L_{X} Q=\{\chi, Q\}=0
$$

where $\chi \in C^{\infty}\left(T^{*} M\right)$ is the symbol or "Hamiltonian" of the vector field $X$. Note that the homological vector field $Q$ is a Jacobi vector field.

Proposition 9. Let $Z \in \operatorname{Vect}(M)$ be a vector field on an odd Jacobi manifold. Then, the following are equivalent:

(1) $Z$ is a Jacobi vector field;

(2) $Z$ is a derivation over the odd Jacobi bracket;

$$
Z\left([f, g]_{J}\right)=[Z(f), g]_{J}+(-1)^{\widetilde{Z}(\tilde{f}+1)}[f, Z(g)]_{J}
$$

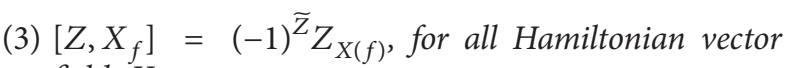
fields $X_{f}$.

Proposition 10. A Hamiltonian vector field $X_{f} \in \operatorname{Vect}(M)$ is a Jacobi vector field if and only if $f \in C^{\infty}(M)$ is Q-closed.

Proposition 11. The assignment $f \leadsto X_{f}$ is a morphism between the odd Lie algebra on $C^{\infty}(M)$ provided by the odd Jacobi brackets and the Lie algebra of vector fields. Specifically, the following holds:

$$
\left[X_{f}, X_{g}\right]=-X_{[f, g]_{J}}
$$

for all $f, g \in C^{\infty}(M)$.

Proposition 12. On an odd Jacobi manifold, the following identity holds:

$$
X_{f g}=(-1)^{\tilde{f}} f X_{g}+(-1)^{\tilde{g}(\tilde{f}+1)} g X_{f}+(-1)^{\tilde{f}+\tilde{g}+1} f g Q .
$$

Remark 13. An even or odd Lie bracket defined on sections of an even line bundle over a manifold which is a first-order differential operator with respect to each argument is known as a Kirillov bracket [16]. Jacobi brackets are Kirillov brackets on trivial line bundles. In this respect, Jacobi brackets serve as a local description of Kirillov brackets but should be seen as a secondary notion. We will not pursue the more general situation of Kirillov brackets here and focus only on Jacobi structures.

\section{Loday-Poisson Brackets}

We are now in a position to state the main theorem of this paper. We draw heavily on the notion of derived brackets in the sense of Kosmann-Schwarzbach; see [2,3] for details.

Theorem 14. Let $(M, S, Q)$ be an odd Jacobi manifold; then, $C^{\infty}(M)$ comes equipped with a canonical Loday-Poisson bracket.

Proof. We prove the theorem by direct construction of the Loday-Poisson bracket. The bracket is canonical up to minor 
issues of conventions. Following Kosmann-Schwarzbach [2], we define the Loday-Poisson bracket as

$$
\{f, g\}_{J}:=(-1)^{\tilde{f}+1}[Q(f), g]_{J} .
$$

The vector field $Q$ is homological and Jacobi. This fact together with the Jacobi identity for the odd Jacobi bracket implies that the Loday-Poisson is even and satisfies the Jacobi-Loday identity. For completeness, we outline the steps here and urge the reader to consult [2].

From the definitions and the Jacobi identity for the odd Jacobi bracket, we have

$$
\begin{aligned}
\left\{f,\{g, h\}_{J}\right\}_{J}=(-1)^{\tilde{f}+\widetilde{g}}\left[Q(f),[Q(g), h]_{J}\right]_{J} \\
=(-1)^{\tilde{f}+\widetilde{g}}\left(\left[\left[[[Q(f), Q(g)]]_{J}, h\right]\right]_{J}\right. \\
\left.+(-1)^{\tilde{f} \widetilde{g}}\left[\left[Q(g),[[Q(f), h]]_{J}\right]\right]_{J}\right) .
\end{aligned}
$$

Using the fact that the homological vector field is a Jacobi vector field, we have

$$
\begin{aligned}
\left\{f,\{g, h\}_{J}\right\}_{J} & \\
= & (-1)^{\tilde{g}+\tilde{f}+1}\left[(-1)^{\tilde{f}+1} Q\left([Q(f), g]_{J}\right), h\right]_{J} \\
& +(-1)^{\tilde{f} \widetilde{g}+\tilde{f}+\widetilde{g}}\left[Q(g),[Q(f), h]_{J}\right]_{J} \\
& -(-1)^{\tilde{g}}\left[\left[Q^{2}(f), g\right]_{J}, h\right]_{J} .
\end{aligned}
$$

As $Q^{2}=0$, the above gives

$$
\left\{f,\{g, h\}_{J}\right\}_{J}=\left\{\{f, g\}_{J}, h\right\}_{J}+(-1)^{\tilde{f} \tilde{g}}\left\{g,\{f, h\}_{J}\right\}_{J} .
$$

Note, however, that the bracket is not automatically skew symmetric. The Leibniz rule follows from the definitions directly:

$$
\begin{aligned}
\{f, g h\}_{J}= & (-1)^{\tilde{f}+1}[Q(f), g h]_{J}, \\
= & (-1)^{\tilde{f}+1}[Q(f), g]_{J} h \\
& +(-1)^{\tilde{f}+1+\tilde{f} \widetilde{g}} g[Q(f), h]_{J} \\
& -[Q(f), \mathbb{1}]_{J} g h, \\
= & \{f, g\}_{J} h+(-1)^{\tilde{f} \tilde{g}} g\{f, h\}_{J} \\
& -[Q(f), \mathbb{1}]_{J} g h,
\end{aligned}
$$

which follows from the modified Leibniz rule for the odd Jacobi bracket and the definition of the Loday-Poisson bracket. Then, as $[f, \mathbb{1}]_{J}=(-1)^{\tilde{f}} Q(f)$, we have $[Q(f), \mathbb{1}]_{J}=$ $\pm Q(Q(f))=0$ as $Q$ is homological. This could also be shown via direct application of the Jacobi identity for the odd Jacobi bracket. Then,

$$
\{f, g h\}_{J}=\{f, g\}_{J} h+(-1)^{\tilde{f} \widetilde{g}} g\{f, h\}_{J} .
$$

Remark 15. Note that the centre of the odd Jacobi algebra consists entirely of Q-closed functions. This is evident as we have $[[f, \mathbb{1}]]_{J}=(-1)^{\tilde{f}} Q(f)$. Thus, the restriction of the Loday-Poisson bracket to the centre of $\left(C^{\infty}(M),[[\cdot, \cdot]]_{J}\right)$ is identically zero or, in other words, the trivial Poisson bracket. by

In local coordinates, the Loday-Poisson bracket is given

$$
\begin{aligned}
\{f, g\}_{J}=(-1)^{\widetilde{B}(\tilde{f}+1)+1}( & (-1)^{\widetilde{A}} S^{B A} Q^{C} \frac{\partial^{2} f}{\partial x^{C} \partial x^{A}} \\
& +S^{B A} \frac{\partial Q^{C}}{\partial x^{A}} \frac{\partial f}{\partial x^{C}} \\
& \left.-Q^{B} Q^{A} \frac{\partial f}{\partial x^{A}}\right) \frac{\partial g}{\partial x^{B}} .
\end{aligned}
$$

Directly from this local expression, we see that the bracket satisfies the right Leibniz rule and is not skew symmetric, in general. Moreover, the Loday-Poisson bracket is a secondorder differential operator in the first argument. From the definitions, it is clear that

$$
\{f, g\}_{J}-(-1)^{\tilde{f} \tilde{g}}\{g, f\}_{J}=(-1)^{\tilde{f}+1} Q\left([f, g]_{J}\right) .
$$

\section{Some Examples}

In this section, we briefly present four examples of odd Jacobi manifolds and the Loday-Poisson brackets associated with them. These examples are taken straight from [1].

Schouten Manifolds can be considered as odd Jacobi manifolds with the homological vector field being the zero vector. In this case the corresponding Loday-Poisson bracket is also trivial,

$$
\{f, g\}_{J}=0 \text {, }
$$

for all $f, g \in C^{\infty}(M)$. Examples of Schouten manifolds include odd symplectic manifolds, which have found applications in physics via the Batalin-Vilkovisky formalism.

Q-manifolds are understood as odd Jacobi manifolds with the almost Schouten structure being zero. Q-manifolds have found important applications in the Batalin-Vilkovisky formalism along-side Schouten structures, $[17,18]$. The odd Jacobi bracket on a $Q$-manifold is then given by

$$
[f, g]_{Q}=(-1)^{\tilde{f}} Q(f g)=(-1)^{\tilde{f}} Q^{A} \frac{\partial f}{\partial x^{A}} g+f Q^{A} \frac{\partial g}{\partial x^{A}}
$$

The associated Loday-Poisson bracket is thus

$$
\{f, g\}_{Q}=(-1)^{\tilde{f}+1} Q(f) Q(g)=(-1)^{\widetilde{B}(\tilde{f}+1)} Q^{B} Q^{A} \frac{\partial f}{\partial x^{A}} \frac{\partial g}{\partial x^{B}},
$$


which is in fact skew symmetric and thus a genuine Poisson bracket. One can see this directly or from the fact that $Q\left([[f, g]]_{Q}\right)=0$.

Remark 16. There is nothing really new here. In essence, all we have is a Poisson structure (bivector) given by $P=$ $\pm(1 / 2)(\varsigma Q)^{2}$, where $\varsigma: \operatorname{Vect}(M) \rightarrow C^{\infty}\left(\Pi T^{*} M\right)$ is the odd isomorphism between vector fields and "one-vectors." Note that $\varsigma Q$ is now even and the homological property becomes $[[\varsigma Q, \varsigma Q]]=0$, where the bracket here is the canonical Schouten-Nijenhuis bracket. Thus, due to the Leibniz rule, it is clear that $[[P, P]]=0$ and we have a Poisson structure. One could also build higher Poisson structures from $Q$-manifolds in this way.

Recall that a Lie Algebroid $E \rightarrow M$ can be described as a weight one homological vector field on the total space of $\Pi E$. This understanding in terms of graded $Q$-manifolds is attributed to Vaintrob [19]. The weight is assigned as zero to the base coordinates and one to the fibre coordinates. In natural local coordinates $\left(x^{A}, \xi^{\alpha}\right)$, the homological vector field is of the form

$$
Q=\xi^{\alpha} Q_{\alpha}^{A}(x) \frac{\partial}{\partial x^{A}}+\frac{1}{2} \xi^{\alpha} \xi^{\beta} Q_{\beta \alpha}^{\gamma}(x) \frac{\partial}{\partial \xi^{\gamma}} \in \operatorname{Vect}(\Pi E)
$$

The associated weight one odd Jacobi bracket is given by

$$
\begin{aligned}
& {[\phi, \psi]_{E}=(-1)^{\tilde{\phi}}\left(\xi^{\alpha} Q_{\alpha}^{A}(x) \frac{\partial \phi}{\partial x^{A}}\right.} \\
&\left.+\frac{1}{2} \xi^{\alpha} \xi^{\beta} Q_{\beta \alpha}^{\gamma}(x) \frac{\partial \phi}{\partial \xi^{\gamma}}\right) \psi \\
&+\phi\left(\xi^{\alpha} Q_{\alpha}^{A}(x) \frac{\partial \psi}{\partial x^{A}}\right. \\
&\left.+\frac{1}{2} \xi^{\alpha} \xi^{\beta} Q_{\beta \alpha}^{\gamma}(x) \frac{\partial \psi}{\partial \xi^{\gamma}}\right),
\end{aligned}
$$

where $\phi, \psi \in C^{\infty}(\Pi E)$ are "Lie algebroid differential forms." The weight of two-Poisson bracket is given in local coordinates by

$$
\begin{aligned}
\{\phi, \psi\}_{E}= & (-1)^{\widetilde{B} \tilde{\phi}+(\widetilde{B}+1) \tilde{\gamma}} \xi^{\gamma} \xi^{\alpha} Q_{\alpha}^{B} Q_{\gamma}^{A} \frac{\partial \phi}{\partial x^{A}} \frac{\partial \psi}{\partial x^{B}} \\
& +\frac{1}{2} \xi^{\gamma} \xi^{\delta} \xi^{\alpha}\left((-1)^{\widetilde{B}(\widetilde{\phi}+1)+(\widetilde{\gamma}+\widetilde{\delta})(\widetilde{B}+1)}\right. \\
& \times Q_{\alpha}^{B} Q_{\delta \gamma}^{\epsilon} \frac{\partial \phi}{\partial \xi^{\epsilon}} \frac{\partial \psi}{\partial x^{B}} \\
& +(-1)^{(\widetilde{\epsilon}+1)(\widetilde{\phi}+1)+\widetilde{\epsilon}(\tilde{\gamma}+1)} \\
& \left.\times Q_{\alpha \delta}^{\epsilon} Q_{\gamma}^{B} \frac{\partial \phi}{\partial x^{B}} \frac{\partial \psi}{\partial \xi^{\epsilon}}\right) \\
& +(-1)^{(\widetilde{\beta}+1)(\widetilde{\phi}+1)+\widetilde{\beta}(\widetilde{\epsilon}+\tilde{\rho})} \\
& \times \frac{1}{4} \xi^{\epsilon} \xi^{\rho} \xi^{\gamma} \xi^{\delta} Q_{\delta \gamma}^{\beta} Q_{\rho \epsilon}^{\alpha} \frac{\partial \phi}{\partial \xi^{\alpha}} \frac{\partial \psi}{\partial \xi^{\beta}} .
\end{aligned}
$$

Remark 17. The constructions here directly generalize to $L_{\infty^{-}}$ algebroids, understood as the pair $(\Pi E, Q)$, where $Q \in$ $\operatorname{Vect}(\Pi E)$ is a homological vector field now inhomogeneous in weight.

Consider the manifold $M=\Pi T^{*} N \times \mathbb{R}^{0 \mid 1}$, where $N$ is a pure even classical manifold. Let us equip this manifold with natural local coordinates $\left(x^{a}, x_{a}^{*}, \tau\right)$, where $\left(x_{a}^{*}\right)$ are fibre coordinates on $\Pi T^{*} N$, which are Grassmann odd and $\tau$ is the coordinate on the factor $\mathbb{R}^{0 \mid 1}$. The manifold $M$ is an odd contact manifold. That is, it comes with an odd contact form given by

$$
\alpha=d \tau-x_{a}^{*} d x^{a} .
$$

Associated with this odd contact form is an odd Jacobi structure given by

$$
\begin{gathered}
S=p_{*}^{a}\left(p_{a}+x_{a}^{*} \pi\right) \in C^{\infty}\left(T^{*} M\right), \\
Q=-\frac{\partial}{\partial \tau} \in \operatorname{Vect}(M),
\end{gathered}
$$

where we have employed fibre coordinates $\left(p_{a}, p_{*}^{a}, \pi\right)$ on $T^{*} M$. The corresponding odd Jacobi bracket is given by

$$
\begin{aligned}
{[f, g]_{J}=} & (-1)^{\tilde{f}+1} \frac{\partial f}{\partial x_{a}^{*}} \frac{\partial g}{\partial x^{a}}-\frac{\partial f}{\partial x^{a}} \frac{\partial g}{\partial x_{a}^{*}} \\
& +x_{a}^{*} \frac{\partial f}{\partial x_{a}^{*}} \frac{\partial g}{\partial \tau}-(-1)^{\tilde{f}+1} \frac{\partial f}{\partial \tau} x_{a}^{*} \frac{\partial g}{\partial x_{a}^{*}} \\
& +f \frac{\partial g}{\partial \tau}-(-1)^{\tilde{f}+1} \frac{\partial f}{\partial \tau} g .
\end{aligned}
$$

Then, the Loday-Poisson bracket is given by

$$
\begin{aligned}
\{f, g\}_{J}= & \frac{\partial^{2} f}{\partial x_{a}^{*} \partial \tau} \frac{\partial g}{\partial x^{a}}-(-1)^{\tilde{f}} \frac{\partial^{2} f}{\partial x^{a} \partial \tau} \frac{\partial g}{\partial x_{a}^{*}} \\
& +(-1)^{\tilde{f}} x_{a}^{*} \frac{\partial^{2} f}{\partial x_{a}^{*} \partial \tau} \frac{\partial g}{\partial \tau}+(-1)^{\tilde{f}} \frac{\partial f}{\partial \tau} \frac{\partial g}{\partial \tau} .
\end{aligned}
$$

\section{Hamiltonian Vector Fields}

We now continue this paper with a study of the algebraic relations between the Hamiltonian vector fields with respect to the odd Jacobi bracket and the (even) Loday-Poisson bracket.

Definition 18. Let $f \in C^{\infty}(M)$ be an arbitrary function. The associated Hamiltonian vector field with respect to the LodayPoisson bracket $Y_{f} \in \operatorname{Vect}(M)$ is defined, namely,

$$
Y_{f}(g)=\{f, g\}_{J}
$$

Throughout this section, we will denote Hamiltonian vector fields with respect to the odd Jacobi structure as $X_{f}$ and those with respect to the Loday-Poisson structure as $Y_{f}$ in order to distinguish the two. Note that $\widetilde{X_{f}}=\widetilde{f}+1$ and that $\widetilde{Y_{f}}=\widetilde{f}$. 
Lemma 19. Let $Y_{f}$ be the Hamiltonian vector fields associated with the function $f \in C^{\infty}(M)$ with respect to the LodayPoisson bracket. Then,

$$
Y_{f}=X_{Q(f)}=-\left[Q, X_{f}\right]
$$

where $X_{f}$ is the Hamiltonian vector field associated with $f$ with respect to the odd Jacobi structure.

Proof. From the definitions

$$
\begin{aligned}
Y_{f}(g) & =\{f, g\}_{J}=(-1)^{\tilde{f}+1}[[Q(f), g]]_{J} \\
& =X_{Q(f)}(g)+Q(Q(f)) g,
\end{aligned}
$$

then given that $Q$ is homological we get $Y_{f}=X_{Q(f)}$. Then using Proposition 9. we get $X_{Q(f)}=-\left[Q, X_{f}\right]$ which establishes the lemma.

The above lemma can be viewed as establishing a mild generalization of bi-Hamiltonian systems. In particular, any vector field that is Hamiltonian with respect to the LodayPoisson bracket is also Hamiltonian with respect to the odd Jacobi structure and the Hamiltonians are related directly via the homological field.

Corollary 20. Let $f \in C^{\infty}(M)$ be an even function that satisfies the "classical master equation" $[f, f]_{J}=0$. Then, this implies that $\{f, f\}_{J}=0$. Furthermore, we have $[Q(f), f]_{J}=0$ and $\{f, Q(f)\}_{J}=0$.

The above corollary naturally generalizes the statement that, for classical bi-Hamiltonian systems, both Hamiltonians are in involution with respect to both Poisson structures. Also, note that the Hamiltonian vector fields with respect to the Loday-Poisson bracket only depend on the Qcohomology class of the Hamiltonian function. Specifically, if $f-f^{\prime}=Q(g)$ for for some $g \in C^{\infty}(M)$, then $Y_{f}=Y_{f^{\prime}}$.

Proposition 21. Let $Y_{f}$ be the Hamiltonian vector field associated with the function $f \in C^{\infty}(M)$ with respect to the LodayPoisson bracket. Then,

$$
\left[Q, Y_{f}\right]=0
$$

Proof. From Lemma 19 and the Jacobi identity for the Lie bracket, we have

$$
\left[Q, Y_{f}\right]=-\left[Q,\left[Q, X_{f}\right]\right]=-\frac{1}{2}\left[[Q, Q], X_{f}\right]=0
$$

as $Q$ is a homological vector field.

Lemma 22. Let $Y_{f}$ be the Hamiltonian vector field associated with the function $f \in C^{\infty}(M)$ with respect to the LodayPoisson bracket. Then, $Y_{f}$ is a Jacobi vector field.

Proof. It follows from Lemma 19 that Hamiltonian vector fields with respect to the odd Jacobi bracket are Jacobi if and only if the Hamiltonian function is $Q$-closed. As $Q^{2}=0$ evidently $Y_{f}$ is Jacobi.
Proposition 23. Let $Y_{f}$ and $Y_{g}$ be the Hamiltonian vector fields associated with the functions $f, g \in C^{\infty}(M)$ with respect to the Loday-Poisson bracket. Then,

$$
\left[Y_{f}, Y_{g}\right]=Y_{\{f, g\}_{j}}
$$

Proof. Via direct computation,

$$
\begin{aligned}
{\left[Y_{f}, Y_{g}\right] } & =\left[X_{Q(f)}, X_{Q(g)}\right] \\
& =-X_{[[Q(f), Q(g)]]_{J}}
\end{aligned}
$$

using the properties of Hamiltonian vector fields associated with the odd Jacobi bracket. Then, using

$$
Q\left([Q(f), g]_{J}\right)=(-1)^{\tilde{f}}[Q(f), Q(g)]_{J},
$$

we arrive at

$$
-X_{[Q(f), Q(g)]_{J}}=(-1)^{\tilde{f}+1} X_{Q\left([Q(f), g]_{J}\right)},
$$

which established the proposition.

The above proposition is rather expected and more interesting are the "mixed" commutators of the Hamiltonian vector fields. In particular, are there nice expressions for $X_{\{f, g\}_{J}}$ and $Y_{[f, g]_{J}}$ ?

Proposition 24. Let $X_{g}$ and $Y_{f}$ be the Hamiltonian vector fields associated with the functions $g, f \in C^{\infty}(M)$ with respect to the odd Jacobi bracket and the Loday-Poisson bracket, respectively. Then,

$$
\left[Y_{f}, X_{g}\right]=(-1)^{\tilde{f}} X_{\{f, g\}_{J}}
$$

Proof. The proposition follows from the fact that $Y_{f}$ is a Jacobi vector field, see Lemma 22 and Proposition 9.

Proposition 25. Let $X_{f}$ and $Y_{f}$ be the Hamiltonian vector fields associated with the function $f \in C^{\infty}(M)$ with respect to the odd Jacobi bracket and the Loday-Poisson bracket, respectively. Then,

$$
(-1)^{\tilde{f}+1} Y_{[f, g]_{J}}=X_{\{f, g\}_{J}}+(-1)^{\tilde{f} \widetilde{g}} X_{\{g, f\}_{J}} .
$$

Proof. From Lemma 19, we have

$$
Y_{[f, g]_{J}}=-\left[Q, X_{[f, g]_{J}}\right]=X_{\mathrm{Q}\left([f, g]_{J}\right)}
$$

Then, using (20), and upon multiplication by overall sign factor, we arrive at the above expression.

Corollary 26. With the definitions previously given

(1) $\left[Q, X_{\{f, g\}_{J}}\right]=-\left[Y_{f}, Y_{g}\right]=-Y_{\{f, g\}_{J}}$;

(2) $\left[Q, Y_{[f, g]_{J}}\right]=0$. 
Expressions for higher nested commutators of Hamiltonian vector field can be worked out from the relations given here and the Jacobi identity for the commutator.

The next natural thing to consider in this section is how the Hamiltonian vector field with respect to the LodayPoisson bracket behaves under the product of two functions.

Proposition 27. On an odd Jacobi manifold, the following identity holds:

$$
\begin{aligned}
Y_{f g}= & f Y_{g}+(-1)^{\tilde{f} \tilde{g}} g Y_{f} \\
& +(-1)^{\tilde{f}+1} Q(f)\left(X_{g}-(-1)^{\tilde{g}} g Q\right) \\
& +(-1)^{\tilde{f} \tilde{g}+\tilde{g}+1} Q(g)\left(X_{f}-(-1)^{\tilde{f}} f Q\right) .
\end{aligned}
$$

Proof. First, note from Lemma 19 and Leibniz rule for $Q$ that

$$
Y_{f g}=X_{Q(f g)}=X_{Q(f) g}+(-1)^{\tilde{f}} X_{f Q(g)} .
$$

Then, application of Proposition 12 produces

$$
\begin{aligned}
Y_{f g}= & f X_{Q(g)}+(-1)^{\tilde{f} \tilde{g}} g X_{Q(f)} \\
& +(-1)^{\tilde{f}+1} Q(f)\left(X_{g}-(-1)^{\tilde{g}} g Q\right) \\
& +(-1)^{\tilde{f} \tilde{g}+\tilde{g}+1} Q(g)\left(X_{f}-(-1)^{\tilde{f}} f Q\right),
\end{aligned}
$$

which implies the proposition.
It is easy to verify the "consistency conditions" $Y_{\mathbb{1 g}}=$ $Y_{g}$ and $Y_{f \mathbb{1}}=Y_{f}$. Furthermore, Proposition 27 can be interpreted as "measuring" the violation of the left Leibniz rule of Loday-Poisson bracket. The failure of the left Leibniz rule is of course a direct consequence of the Loday-Poisson bracket being a second-order differential operator in the first argument. Specifically, we have

$$
\begin{aligned}
\{f g, h\}_{J}= & f\{g, h\}_{J}+(-1)^{\tilde{g} \tilde{h}}\{f, h\}_{J} g \\
& +(-1)^{\tilde{f}+1} Q(f)\left((-1)^{\tilde{g}}[g, h]_{J}-Q(g h)\right) \\
& +(-1)^{\tilde{f} \tilde{g}+\tilde{g}+1} Q(g)\left((-1)^{\tilde{f}}[f, h]_{J}-Q(f h)\right) .
\end{aligned}
$$

Remark 28. The Loday-Poisson bracket is then a biderivation if we restrict the left-hand entries of the bracket to be Q-closed. However, this condition implies that the LodayPoisson bracket is trivial. The other extreme is to insist that $(-1)^{\tilde{f}}[[f, h]]_{J}-Q(f h)=0$ for all, $f, h \in C^{\infty}(M)$. This implies that $[[f, h]]_{J}=(-1)^{\tilde{f}} Q(f h)$ and thus the underlying odd Jacobi structure is $(0, Q)$. That is, we have "just" a $Q$-manifold.

The similarity between the relations satisfied by the two classes of Hamiltonian vector field on an odd Jacobi manifold and the Cartan identities is striking, but not surprising as the Cartan calculus can be understood in terms of derived brackets [3]. In essence, we have the associations

Hamiltonian vector fields w.r.t odd Jacobi structure $\longleftrightarrow$ Interior derivative;

Hamiltonian vector fields w.r.t Loday-Poisson structure $\longleftrightarrow$ Lie derivative

Loday-Poisson bracket $\longleftrightarrow$ Lie bracket.

With these formal algebraic similarities in mind, one can interpret the constructions here as a (partially noncommutative) generalization of the Cartan calculus. However, as the interior product cannot directly be understood as a Hamiltonian vector field with respect to some odd Jacobi structure, the Cartan calculus cannot be seen as a special case of the constructions given in this work.

\section{The Derived Product}

Definition 29. Let $(M, Q)$ be a $Q$-manifold. The derived product is the binary operation *: $C^{\infty}(M) \times C^{\infty}(M) \rightarrow$ $C^{\infty}(M)$ defined as

$$
f * g=(-1)^{\tilde{f}+1} Q(f) g,
$$

where $f, g \in C^{\infty}(M)$.

It is easy to verify that this derived product is associative, but not (super) commutative. The derived product is an odd form of noncommutative multiplication on $C^{\infty}(M): \widetilde{f * g}=$ $\widetilde{f}+\tilde{g}+1$. The notion of a derived product is also due to Loday and has its origin in his study of dialgebras [20]. The derived product on a $Q$-manifold can be viewed in the light of deformation quantisation. That is, the vector space structure of the smooth functions on the Q-manifold remains the same; it is only the product that is deformed. Also note that the derived $*$-commutator is given by

$$
[f, g]_{*}=f * g-(-1)^{(\tilde{f}+1)(\widetilde{g}+1)} g * f=-[f, g]_{Q} .
$$

We observe that, up to a sign, the odd Jacobi bracket generated by a homological vector field is the derived * commutator. This is in the same spirit as understanding Poisson brackets as the classical limit of commutators in deformation quantisation. However, note that $\mathbb{1} * f=0$, meaning that constant function $\mathbb{1}$ is not the identity ("barunit" in Loday's language) for the derived product. Also note $f * \mathbb{1}= \pm Q(f)$. Furthermore, we do not have any parameter playing the role of $\hbar$. 
Remark 30. We will not have any course in this work to employ ideas from the theory of dialgebras. We only remark that "dialgebras are to Loday algebra what associative algebra is to Lie algebra." The relation between the *-commutator and the odd Jacobi bracket on a Q-manifold is an example of this.

The derived $*$-commutator has the following easy to verify properties:

(1) $[f, g]_{*}=-(-1)^{(\tilde{f}+1)(\widetilde{g}+1)}[g, f]_{*}$,

(2) $[f, \mathbb{1}]_{*}=-[\mathbb{1}, f]_{*}$, for all $f$,

(3) $[f, g]_{*} * h=0$.

Lemma 31. Let $(M, S, Q)$ be an odd Jacobi manifold. Then, the odd Jacobi bracket satisfies a generalized Leibniz rule given by

$$
\begin{aligned}
{[f, g * h]_{J}=} & {[f, g]_{J} * h } \\
& +(-1)^{(\tilde{f}+1)(\tilde{g}+1)} g *[f, h]_{J} \\
& +f * g * h+(-1)^{\tilde{g}}\{f, g\}_{J} h,
\end{aligned}
$$

where $f, g, h \in C^{\infty}(M)$.

Proof. Direct from the definitions and the modified Leibniz rule for the odd Jacobi bracket, we have

$$
\begin{aligned}
{[f, g * h]_{J}=} & (-1)^{\tilde{g}+1}[f, Q(g) h]_{J} \\
= & (-1)^{\widetilde{g}+1}[f, Q(g)]_{J} h \\
& +(-1)^{\tilde{g}+1+(\widetilde{g}+1)(\tilde{f}+1)} Q(g)[f, h]_{J} \\
& -(-1)^{\widetilde{g}+1}[f, \mathbb{1}]_{J} Q(g) h .
\end{aligned}
$$

Then, using the fact that the homological vector field $Q$ is a Jacobi vector field the above can be cast in the form

$$
\begin{aligned}
{[f, g * h]_{J}=} & (-1)^{\tilde{f}+\tilde{g}} Q\left([f, g]_{J}\right) h \\
& -(-1)^{\tilde{f}+\tilde{g}}[Q(f), g]_{J} h \\
& +(-1)^{\tilde{f}(\tilde{g}+1)} Q(g)[f, h]_{J} \\
& +(-1)^{\tilde{f}+\tilde{g}}[\mathbb{1}, f]_{J} Q(g) h .
\end{aligned}
$$

Then, using the definitions, the lemma is established.

Proposition 32. Let $(M, Q)$ be a $Q$-manifold. The *-commutator is a Schouten bracket with respect to the derived product.

Proof. The skew symmetry follows from Definition 29. The appropriate Jacobi identity follows directly from the equivalence of the $*$-commutator with the odd Jacobi bracket derived from the homological vector field. Thus, the *commutator gives an odd Lie bracket. Only the Leibniz rule is not immediate. However, this follows from Lemma 31, noting that for the case in hand $(-1)^{\widetilde{g}}\{f, g\}_{Q} h=$ $(-1)^{\tilde{f}+\tilde{g}+1} Q(f) Q(g) h=-f * g * h$.
The above proposition is the direct odd parallel of the well-known result that a standard commutator on a (possibly) noncommutative algebra is in fact a Poisson bracket. Indeed, one could ignore the relation between odd noncommutative product and odd Jacobi brackets and establish the Jacobi identity directly from the definition.

Theorem 33. Let $(M, S, Q)$ be an odd Jacobi manifold. Then, the Loday-Poisson bracket obeys the Leibniz rule with the derived product:

$$
\{f, g * h\}_{J}=\{f, g\}_{J} * h+(-1)^{\tilde{f}(\tilde{g}+1)} g *\{f, h\}_{J},
$$

where $f, g, h \in C^{\infty}(M)$.

Proof. The above theorem follows directly from Lemma 31, upon the replacement $f \rightarrow Q(f)$ and the definition of the Loday-Poisson bracket.

Statement. Theorems 14 and 33 tell us that not only does the Loday-Poisson bracket on an odd Jacobi bracket obey the right Leibniz rule over the standard product of smooth functions but also for the odd derived product.

Corollary 34. Directly from the above theorem, we get the mixed Loday-Jacobi identity

$$
\left\{f,[g, h]_{*}\right\}_{J}=\left[\{f, g\}_{J}, h\right]_{*}+(-1)^{\tilde{f}(\widetilde{g}+1)}\left[g,\{f, h\}_{J}\right]_{*},
$$

where $f, g, h \in C^{\infty}(M)$.

Proposition 35. With the definitions previously given

$$
\begin{aligned}
& \text { (1) } X_{f * g}=(-1)^{\tilde{f}+1}(f * \mathbb{1}) X_{g}+(-1)^{\tilde{f} \widetilde{g}} g X_{(f * \mathbb{1})}+ \\
& (-1)^{\tilde{f}+\tilde{g}}(f * g) Q, \\
& \text { (2) } Y_{f * g}=(f * \mathbb{1}) Y_{g}+(-1)^{(\tilde{f}+1)(\widetilde{g}+1)}(g * \mathbb{1}) Y_{f}-(f * g * \mathbb{1}) Q, \\
& \text { where } f, g, h \in C^{\infty}(M) .
\end{aligned}
$$

Proof. The proof follows the definitions directly.

(1) From Proposition 12, we have

$$
\begin{aligned}
X_{Q(f) g}= & (-1)^{\tilde{f}+1} Q(f) X_{g}+(-1)^{\tilde{f} \tilde{g}} g X_{Q(f)} \\
& +(-1)^{\tilde{f}+\tilde{g}} Q(f) g Q .
\end{aligned}
$$

Then, using the definition of the derived product and $f * \mathbb{1}=(-1)^{\tilde{f}+1} Q(f)$, the first part of the proposition is established.

(2) From Proposition 27, we have

$$
\begin{aligned}
Y_{Q(f) g}= & Q(f) Y_{g}+(-1)^{(\tilde{f}+1) \tilde{g}+\tilde{g}+1} \\
& \times Q(g)\left(X_{Q(f)}+(-1)^{\tilde{f}} Q(f) Q\right),
\end{aligned}
$$


taking into account that $Q^{2}=0$. Then, multiplying by the correct sign factor and using the definition of $Y_{f}$ produce

$$
\begin{aligned}
Y_{f * g}= & (f * 1) Y_{g}+(-1)^{(\tilde{f}+1)(\tilde{g}+1)}(g * 1) Y_{f} \\
& +(-1)^{\tilde{f} \tilde{g}} Q(g) Q(f) Q .
\end{aligned}
$$

Then, using $Q(g) Q(f)=(-1)^{(\tilde{f}+1)(\tilde{g}+1)} Q(f) Q(g)$ and the definition of the derived product, the second part of the proposition is established.

Remark 36. As far as the author is aware, the case of Poissonlike brackets on algebra with an odd form of multiplication has not been studied in detail.

\section{Application to Jacobi Algebroids}

An interesting class of odd Jacobi manifolds is the Jacobi algebroids $[13,21]$. We apply some of the previous constructions to the setting of Jacobi algebroids.

Definition 37. A vector bundle $E \rightarrow M$ is said to have the structure of a Jacobi algebroid if and only if the total space of $\Pi E^{*}$ comes equipped with a weight minus one odd Jacobi structure.

It is well known that Jacobi algebroids, which are also known as generalized Lie algebroids, are equivalent to Lie algebroids in the presence of a 1-cocycle; see [21]. Let us employ natural local coordinates $\left(x^{A}, \eta_{\alpha}, p_{A}, \pi^{\alpha}\right)$ on the total space of $T^{*}\left(\Pi E^{*}\right)$. The weight is assigned as $w\left(x^{A}\right)=0$, $w\left(p_{A}\right)=0, w\left(\eta_{\alpha}\right)=+1$, and $w\left(\pi^{\alpha}\right)=-1$. This is the natural weight associated with the vector bundle structure $E^{*} \rightarrow M$.

The parity of the coordinates is given by $\tilde{x}^{A}=\widetilde{A}, \widetilde{\eta}_{\alpha}=$ $(\widetilde{\alpha}+1), \widetilde{p}_{A}=\widetilde{A}$, and $\tilde{\pi}^{\alpha}=(\widetilde{\alpha}+1)$. In these natural local coordinates, the odd Jacobi structure is given by

$$
\begin{gathered}
S=(-1)^{\tilde{\alpha}} \pi^{\alpha} Q_{\alpha}^{A}(x) p_{A}+(-1)^{\tilde{\alpha}+\tilde{\beta}} \frac{1}{2} \pi^{\alpha} \pi^{\beta} Q_{\beta \alpha}^{\gamma} \eta_{\gamma}, \\
Q=\pi^{\alpha} Q_{\alpha}(x),
\end{gathered}
$$

which are both functions on the total space of $T^{*}\left(\Pi E^{*}\right)$. The algebra of "multivector fields" $C^{\infty}\left(\Pi E^{*}\right)$ comes equipped with an odd Jacobi bracket, namely,

$$
\begin{aligned}
{[X, Y]_{E}=} & (-1)^{\widetilde{X}+1}\left\{\{S, X\}_{T^{*}\left(\Pi E^{*}\right)}, Y\right\}_{T^{*}\left(\Pi E^{*}\right)} \\
& -(-1)^{\widetilde{X}+1}\{\mathcal{Q}, X Y\}_{T^{*}\left(\Pi E^{*}\right)},
\end{aligned}
$$

with $X, Y \in C^{\infty}\left(\Pi E^{*}\right)$.
In natural local coordinates, this bracket is given by

$$
\begin{aligned}
{[X, Y]_{E}=} & Q_{\alpha}^{A}\left((-1)^{(\widetilde{X}+\widetilde{\alpha}+1)(\widetilde{A}+1)} \frac{\partial X}{\partial \eta_{\alpha}} \frac{\partial Y}{\partial x^{A}}\right. \\
& \left.-(-1)^{(\widetilde{X}+1) \widetilde{\alpha}} \frac{\partial X}{\partial x^{A}} \frac{\partial Y}{\partial \eta_{\alpha}}\right) \\
& -(-1)^{(\widetilde{X}+1) \widetilde{\alpha}+\widetilde{\beta}} Q_{\alpha \beta}^{\gamma} \eta_{\gamma} \frac{\partial X}{\partial \eta_{\beta}} \frac{\partial Y}{\partial \eta_{\alpha}} \\
& +(-1)^{\widetilde{X}} Q_{\alpha} \frac{\partial X}{\partial \eta_{\alpha}} Y+X Q_{\alpha} \frac{\partial Y}{\partial \eta_{\alpha}}
\end{aligned}
$$

where $X=X(x, \eta)=X(x)+X^{\alpha}(x) \eta_{\alpha}+(1 / 2 !) X^{\alpha \beta}(x) \eta_{\beta} \eta_{\alpha}+\cdots$ and so forth. Clearly, this odd Jacobi bracket is of weight minus one. If $Q_{\alpha}=0$, then the Jacobi algebroid reduces to a genuine Lie algebroid and the above bracket is a weight minus one Schouten bracket. The weight minus one odd Jacobi bracket is a natural generalization of the weight minus one Schouten bracket associated with a Lie algebroid.

Now, let us proceed to the Loday-Poisson bracket derived from the weight minus one odd Jacobi bracket and the homological vector field $Q=Q_{\alpha}\left(\partial / \partial \eta_{\alpha}\right)$. In natural local coordinates, the Loday-Poisson bracket is given by

$$
\begin{aligned}
\{X, Y\}_{E}=Q_{\alpha}^{A}( & (-1)^{\widetilde{A}(\widetilde{X}+\widetilde{\alpha})} Q_{\delta} \frac{\partial^{2} X}{\partial \eta_{\delta} \partial \eta_{\alpha}} \frac{\partial Y}{\partial x^{A}} \\
& +(-1)^{\widetilde{X}(\widetilde{\alpha}+1)} \frac{\partial Q_{\delta}}{\partial x^{A}} \frac{\partial X}{\partial \eta_{\delta}} \frac{\partial Y}{\partial \eta_{\alpha}} \\
& \left.+(-1)^{\widetilde{X}(\widetilde{\alpha}+1)+\widetilde{A}} Q_{\delta} \frac{\partial^{2} X}{\partial \eta_{\delta} \partial x^{A}} \frac{\partial Y}{\partial \eta_{\alpha}}\right) \\
& -(-1)^{\widetilde{X}(\widetilde{\alpha}+1)} Q_{\alpha \beta}^{\gamma} \eta_{\gamma} Q_{\delta} \frac{\partial^{2} X}{\partial \eta_{\delta} \partial \eta_{\beta}} \frac{\partial Y}{\partial \eta_{\alpha}} \\
& +(-1)^{\widetilde{\alpha}(\widetilde{X}+1)} Q_{\alpha} Q_{\beta} \frac{\partial X}{\partial \eta_{\beta}} \frac{\partial Y}{\partial \eta_{\alpha}} .
\end{aligned}
$$

By construction, which is easily verified in natural local coordinates, the associated Loday-Poisson bracket is of weight minus two.

Remark 38. The Loday-Poisson bracket on $C^{\infty}\left(\Pi E^{*}\right)$ should not be confused with the Poisson bracket on $C^{\infty}\left(E^{*}\right)$ associated with the Lie algebroid structure "behind" the Jacobi algebroid. Indeed, if we have a Lie algebroid and the trivial 1-cocycle $Q=0$, then the associated Loday-Poisson bracket is obviously itself trivial.

The algebra of "multivector fields" $C^{\infty}\left(\Pi E^{*}\right)$ comes equipped with a derived product, namely,

$$
X * Y=(-1)^{\widetilde{X}+1} Q(X) Y=(-1)^{\widetilde{X}+1} Q_{\alpha} \frac{\partial X}{\partial \eta_{\alpha}} Y .
$$


Note that $f * X=0$, where $f \in C^{\infty}(M)$. Via Theorem 33, the Loday-Poisson bracket satisfies the Leibniz rule from the left over the derived product:

$$
\{X, Y * Z\}_{E}=\{X, Y\}_{E} * Z+(-1)^{\widetilde{X}(\widetilde{Y}+1)} Y *\{X, Z\}_{E},
$$

where $X, Y$, and $Z \in C^{\infty}\left(\Pi E^{*}\right)$.

Statement. "Multivector fields" on a Jacobi algebroid can be considered as elements of a noncommutative Loday-Poisson algebra with an odd form of multiplication.

\section{Final Remarks}

In this paper, we used the derived bracket formalism to construct a Loday bracket on $C^{\infty}(M)$ from the initial datum of an odd Jacobi structure $(S, Q)$ on the supermanifold $M$. The Loday bracket in question is the bracket derived from the odd Jacobi bracket and the homological vector field $Q$. Furthermore, it was shown that this Loday bracket satisfies the Leibniz rule acting to the right over the standard supercommutative product of functions and the derived product generated by the homological vector field. Thus, we employ the nomenclature right Loday-Poisson bracket. Some of the relations between the various Hamiltonian vector fields were also explored, as were some specific examples of LodayPoisson brackets such as those in the theory of Lie algebroids and Jacobi algebroids.

Is it important to remark that the construction of the Loday-Poisson bracket from the odd Jacobi bracket presented here makes use of only half the structure available, namely, just the homological vector field. This is the obvious thing to do if one wants to pass from an odd Jacobi bracket to an even Loday bracket. A natural question here is as follows: can one do better and use the full odd Jacobi structure to pass from the odd Jacobi bracket to an even Loday bracket?

\section{Conflict of Interests}

The author declares that there is no conflict of interests regarding the publication of this paper.

\section{Acknowledgments}

The author would like to thank Professor J. Grabowski and Dr. R. A. Mehta for their comments on earlier drafts of this work. The author must also thank the anonymous referees for their invaluable comments and suggestions that have greatly improved the presentation of this work. The author graciously acknowledges the support of the Warsaw Center of Mathematics and Computer Science.

\section{References}

[1] A. J. Bruce, "Odd Jacobi manifolds: general theory and applications to generalised Lie algebroids," Extracta Mathematicae, vol. 27, no. 1, pp. 91-123, 2012.
[2] Y. Kosmann-Schwarzbach, "From Poisson algebras to Gerstenhaber algebras," Annales de l'Institut Fourier, vol. 46, no. 5, pp. 1243-1274, 1996.

[3] Y. Kosmann-Schwarzbach, "Derived brackets," Letters in Mathematical Physics, vol. 69, pp. 61-87, 2004.

[4] J.-L. Loday, Cyclic Homology, Springer, Berlin, Germany, 1992, 2nd edition: 1998.

[5] J.-L. Loday, "Une version non commutative des algèbres de Lie: les algèbres de Leibniz," L'Enseignement Mathématique, vol. 39, no. 3-4, pp. 269-293, 1993.

[6] J. Grabowski and G. Marmo, "Non-antisymmetric versions of Nambu-Poisson and algebroid brackets," Journal of Physics A, vol. 34, no. 18, pp. 3803-3809, 2001.

[7] J. Grabowski and G. Marmo, "Binary operations in classical and quantum mechanics," in Classical and Quantum Integrability, J. Grabowski and P. Urbański, Eds., vol. 59, pp. 163-172, Banach Center, Warsaw, Poland, 2003.

[8] J. M. Casas and T. Datuashvili, "Noncommutative LeibnizPoisson algebras," Communications in Algebra, vol. 34, no. 7, pp. 2507-2530, 2006.

[9] T. Voronov, "Graded manifolds and Drinfeld doubles for Lie bialgebroids," in Quantization, Poisson Brackets and Beyond, vol. 315 of Contemporary Mathematics, pp. 131-168, American Mathematical Society, Providence, RI, USA, 2002.

[10] J.-L. Koszul, "Crochet de Schouten-Nijenhuis et cohomologie," in The Mathematical Heritage of Élie Cartan, Numero Hors, pp. 257-271, Astérisque, Lyon, France, 1985.

[11] A. Lichnerowicz, "Les variétés de Poisson et leurs algèbres de Lie associées," Journal of Differential Geometry, vol. 12, no. 2, pp. 253-300, 1977.

[12] A. Lichnerowicz, "Les variétés de Jacobi et leurs algèbres de Lie associées," Journal de Mathématiques Pures et Appliquées, vol. 57, no. 4, pp. 453-488, 1978.

[13] J. Grabowski and G. Marmo, "Jacobi structures revisited," Journal of Physics A, vol. 34, no. 49, pp. 10975-10990, 2001.

[14] J. Grabowski and G. Marmo, "The graded Jacobi algebras and (co)homology," Journal of Physics A, vol. 36, no. 1, pp. 161-181, 2003.

[15] J. Grabowski, "Graded contact manifolds and contact Courant algebroids," Journal of Geometry and Physics, vol. 68, pp. 27-58, 2013.

[16] A. A. Kirillov, "Local Lie algebras," Uspekhi Matematicheskikh Nauk, vol. 31, no. 4, pp. 57-76, 1976 (Russian).

[17] M. Alexandrov, M. Kontsevich, A. Schwarz, and O. Zaboronsky, "The geometry of the master equation and topological quantum field theory," International Journal of Modern Physics A, vol. 12, no. 7, pp. 1405-1430, 1997.

[18] A. Schwarz, "Semiclassical approximation in Batalin-Vilkovisky formalism," Communications in Mathematical Physics, vol. 158, no. 2, pp. 373-396, 1993.

[19] A. Y. Vaŭntrob, "Lie algebroids and homological vector fields," Russian Mathematical Surveys, vol. 52, no. 2, pp. 428-429, 1997.

[20] J.-L. Loday, "Dialgebras," in Dialgebras and Related Operads, vol. 1763 of Lecture Notes in Mathematics, pp. 7-66, Springer, Berlin, Germany, 2001.

[21] D. Iglesias and J. C. Marrero, "Generalized Lie bialgebroids and Jacobi structures," Journal of Geometry and Physics, vol. 40, no. 2, pp. 176-199, 2001. 


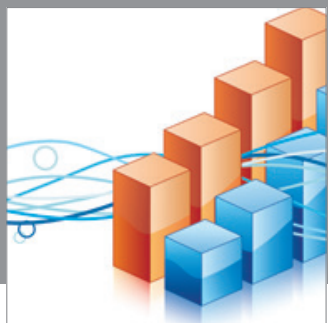

Advances in

Operations Research

mansans

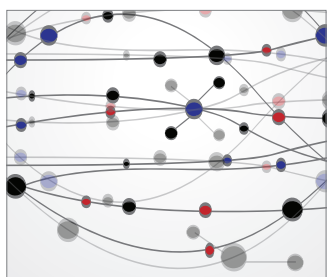

The Scientific World Journal
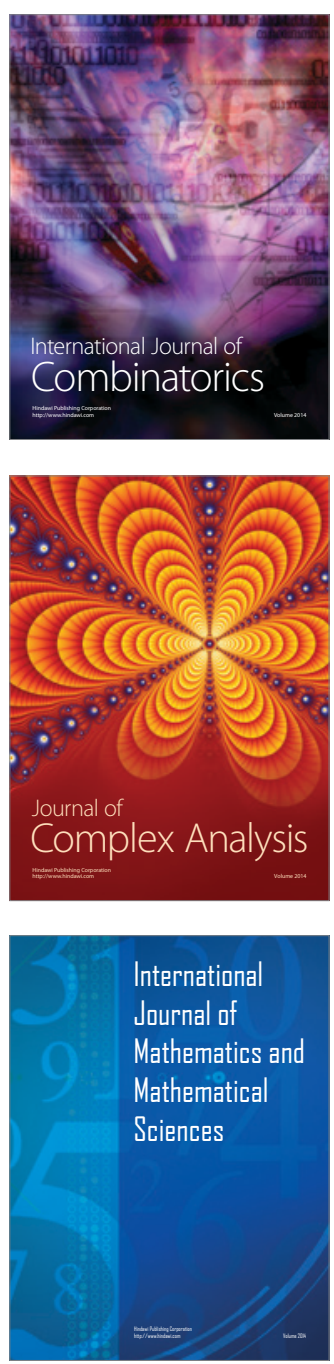
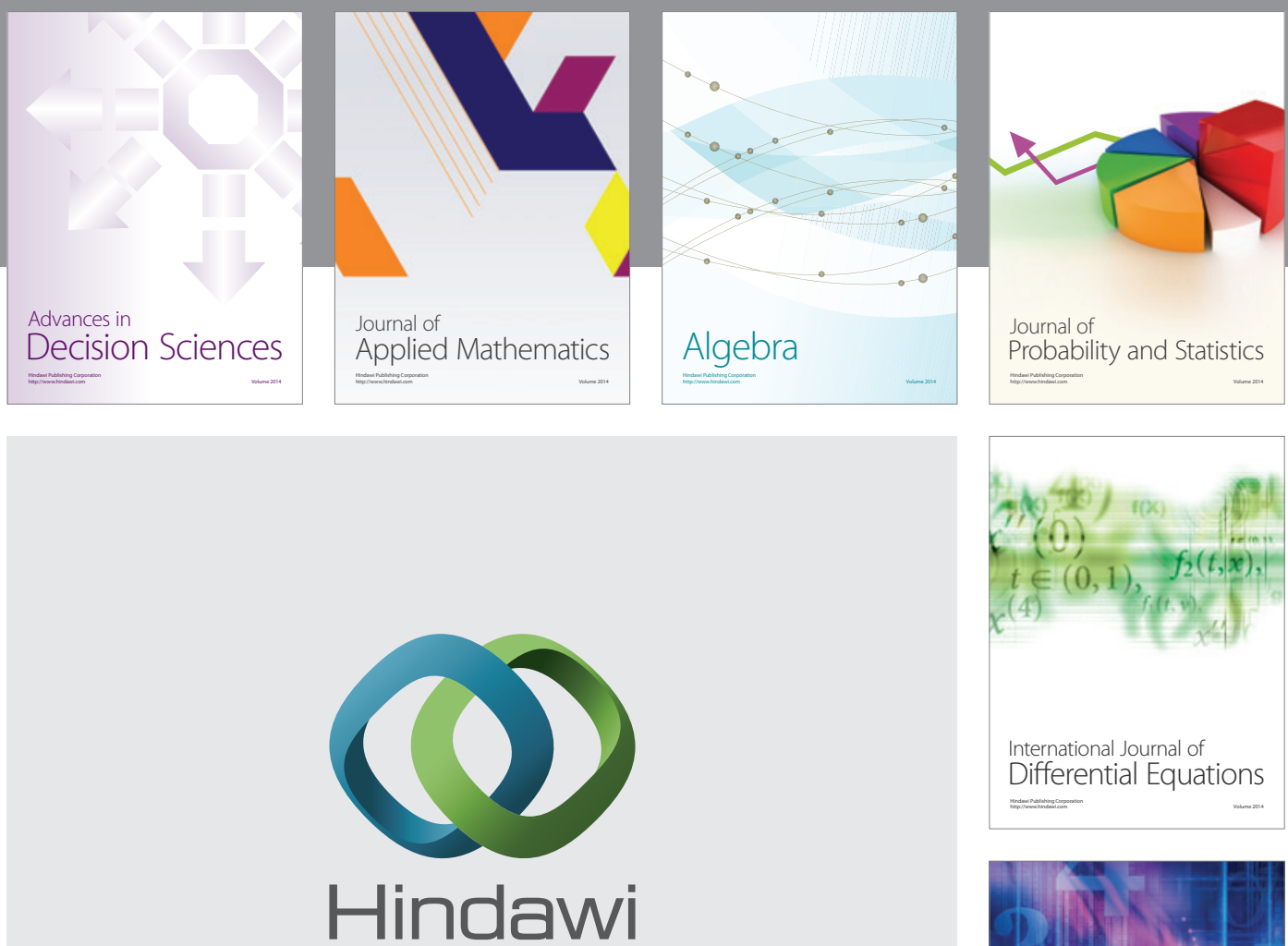

Submit your manuscripts at http://www.hindawi.com
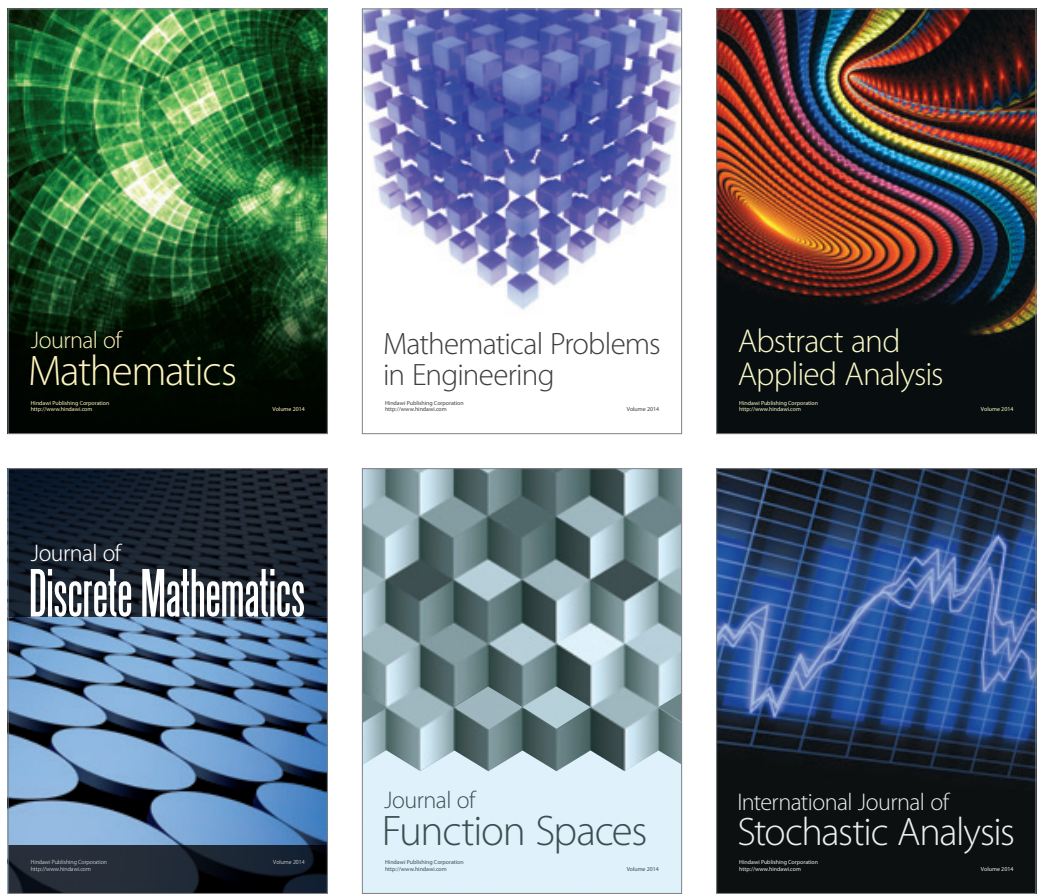

Journal of

Function Spaces

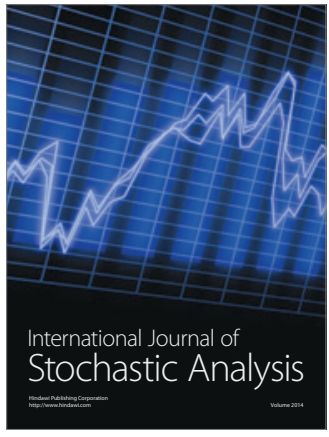

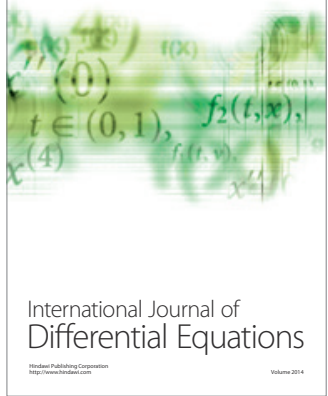
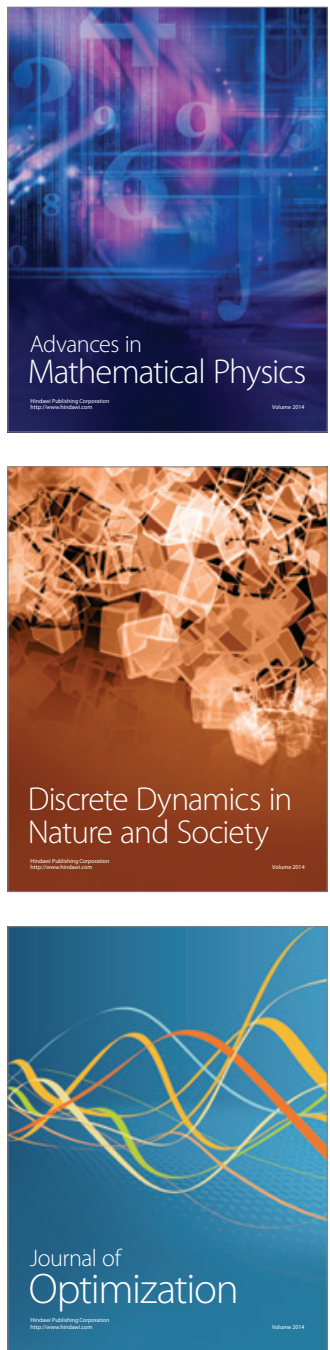